\title{
Avner Baz' Kritik an der Methode möglicher Fälle
}

Avner Baz argumentiert in den Kapiteln 3 bis 6 von When Words Are Called For und in zwei Artikeln, die 2016 erschienen sind, gegen die Brauchbarkeit der Methode möglicher Fälle. ${ }^{1}$ Der Fokus seiner Argumentation liegt auf der Frage des Epistemologen (»theorist's question«): der Frage, die Epistemologinnen und Epistemologen in Bezug auf von ihnen konstruierte Gedankenexperimente stellen. Diese Frage hat die Form »Weiß $S$, dass $p$ ? «, und ihre korrekte Beantwortung soll uns Aufschluss darüber geben, was Wissen ist beziehungsweise welchen Begriff des Wissens wir haben. Bei Baz lassen sich zwei Argumente gegen die Brauchbarkeit dieser Methode identifizieren:

1. Fragen der Form »Weiß $S$, dass $p$ ? «, die wir im Alltag stellen, haben nie eine Bedeutung der Art, wie sie Epistemologinnen und Epistemologen vorschwebt. Auch wenn wir uns vorstellen können, uns im echten Leben in einem Gettier-Fall wiederzufinden, entspräche keine Frage der Form »Weiß $S$, dass $p$ ? «, die wir in einer solchen Situation stellen könnten, der Frage des Epistemologen. Dies ist aus dem folgenden Grund relevant: Verteidigerinnen und Verteidiger der Methode möglicher Fälle begründen unsere Kompetenz in der Beantwortung der Frage des Epistemologen damit, dass die einzige Fähigkeit, die wir für diese Beantwortung benötigen, eine ist, von der wir im Alltag ständig Gebrauch machen. Nämlich die Fähigkeit, den Ausdruck »wissen« auf Einzelfälle anzuwenden beziehungsweise Fälle einzuteilen in Fälle von Wissen und Fälle von Nicht-Wissen. Da wir in diesen Dingen so geübt sind, so die Idee, können wir uns auf unsere Urteile in Bezug auf mögliche Fälle von Wissen oder Nicht-Wissen verlassen, und folglich sind unsere Reaktionen auf die Frage des Epistemologen als Evidenz dafür brauchbar, welchen Wissensbegriff wir haben. Sollte Baz damit Recht haben, dass nichts von dem, was wir im Alltag machen, der Beantwortung der Frage des Epistemologen ähnlich ist, dann können wir nicht mehr unter Rückgriff auf die oben genannte Begründung geltend machen, dass wir dazu fähig sind, die Frage des Epistemologen zu beantworten. Wir bräuchten

1 Auch in seinem 2017 erschienenen The Crisis of Method in Contemporary Analytic Philosophy befasst er sich wieder unter anderem mit der Methode möglicher Fälle. 
also ein neues Argument dafür, dass unseren Reaktionen auf diese Frage ein evidenzieller Wert zukommt für die Analyse unseres Wissensbegriffs.

2. Die Frage des Epistemologen hat keine Bedeutung. Die Bedeutung von Äußerungen der Form »Weiß $S$, dass $p$ ? « beziehungsweise $\gg S$ weiß, dass $p$ « hängt wesentlich vom Witz solcher Äußerungen ab. Die Frage des Epistemologen hat keinen Witz, der dem Ausdruck »Weiß $S$, dass $p$ ?« eine Bedeutung verleihen könnte.

Als Alternative zur Methode möglicher Fälle schlägt Baz ein Vorgehen vor, welches stark der im Kapitel 2 des vorliegenden Buchs beschriebenen Methode typischer Fälle ähnelt: Wenn wir uns klar darüber werden wollen, was das Wort »wissen « bedeutet, sollten wir uns, so Baz, überlegen, welches die Funktion dieses Wortes ist. Und zu diesem Zweck sollten wir verschiedene Kontexte durchgehen, in denen es natürlich ist, das Wort »wissen « zu verwenden, und uns jeweils die Frage stellen, was das Wort in diesen Kontexten leistet. ${ }^{2}$ Ich stimme Baz darin zu, dass diese Methode im Kontext einer Analyse unseres Wissensbegriffs hilfreich ist. Nicht einverstanden bin ich dagegen mit seinen oben zusammengefassten Argumenten gegen die Brauchbarkeit der Methode möglicher Fälle. Im vorliegenden Kapitel rekonstruiere ich beide Argumente von Baz im Detail und versuche zu zeigen, dass keines von ihnen stichhaltig ist. Ein wichtiger Aspekt stellt in diesem Zusammenhang Baz' Position zur Mehrdeutigkeit von $\gg S$ weiß, dass $p$ « dar, die ich, um sie besser verständlich zu machen, von der Auffassung von Gerhard Ernst abgrenzen werde. Zum Schluss des Kapitels werde ich mich mit einigen Argumenten gegen die Stichhaltigkeit von Ernsts Argumentation befassen.

\section{2 Stellt sich die Frage des Epistemologen für uns im Alltag?}

Ausgangspunkt von Baz' erstem Argument gegen die Brauchbarkeit der Methode möglicher Fälle sind zwei Einwände gegen die Idee, dass es zur Beantwortung der Frage, was Wissen ist, hilfreich ist, verschiedene Situationen hinsichtlich der Frage zu beurteilen, ob ein Akteur in diesen Situationen (in Bezug auf eine bestimmte Proposition) über Wissen verfügt oder nicht. Der erste dieser Einwände stammt Baz zufolge ursprünglich von Stephen Stich und bezieht sich auf die unter anderem von Vertreterinnen und Vertretern der Experimentellen Philosophie aufgedeckten, interpersonellen und interkulturellen Unterschiede in den Reaktionen auf Gedankenexperimente: Wieso sollten wir die Intuitionen, die wir selbst in Bezug auf das Vorliegen

2 Vgl. Baz 2012, S. 45, 139, 187. 
von Wissen in bestimmten Situationen haben, gegenüber den Intuitionen anderer Personen bevorzugt behandeln? Der zweite Einwand ist prinzipiell gegen die Nützlichkeit von Intuitionen bei der Analyse von Begriffen gerichtet: Wieso sollten wir unseren Intuitionen in Bezug auf mögliche Fälle überhaupt ein Gewicht zuschreiben, wenn es darum geht, herauszufinden, ob in einer gegebenen Situation tatsächlich Wissen vorliegt oder nicht? Welchen Anhaltspunkt haben wir dafür, dass die Intuitionen, die wir in Reaktion auf die Frage des Epistemologen herausbilden, korrekt sind? Als Urheber dieses zweiten Einwands identifiziert Baz Robert Cummins. ${ }^{3}$ Der zweite Einwand bliebe selbst dann bestehen, wenn alle Leute dieselben Intuitionen in Bezug auf mögliche Fälle hätten. Aber wenn wir die Befunde der Experimentellen Philosophie ernst nehmen können und es tatsächlich große interpersonelle und interkulturelle Unterschiede in den Reaktionen auf Gedankenexperimente gibt, drängt sich der zweite Einwand umso stärker auf: Die Befunde der Experimentellen Philosophie können als Hinweise dafür betrachtet werden, dass auf unsere Intuitionen in Bezug auf Gedankenexperimente kein Verlass ist.

Im Sinne einer möglichen Erwiderung auf diese Einwände bespricht Baz ein Argument von Timothy Williamson: Es gibt überhaupt keinen Grund, im Zusammenhang mit unserer Beurteilung von Gedankenexperimenten von »Intuitionen« zu sprechen: Worauf wir uns stützen, wenn wir die Frage beantworten, ob in einer bestimmten Situation Wissen vorliegt oder nicht, ist unsere alltägliche Kompetenz in der Klassifikation von Fällen:Wenn ich einem Akteur in einem Gettier-Fall Wissen abspreche, dann basiert dieses Urteil ebenso wenig auf einer Intuition, wie wenn ich im Alltag beispielsweise über meinen Nachbarn sage, er wisse nicht, dass ich in den Ferien sei. ${ }^{4}$ Der zweite oben genannte Einwand erübrigt sich damit. Sofern wir nicht in Zweifel ziehen wollen, dass wir im Alltag regelmäßig dazu in der Lage sind, Fälle von Wissen als solche zu erkennen, sind wir darin gerechtfertigt, unsere Klassifikation erfundener Fälle in der Philosophie als Hinweise darauf ernst zu nehmen, in welchen Fällen Wissen vorliegt und in welchen nicht. ${ }^{5}$ Dies bedeutet nicht, dass wir mit unseren Klassifikationen immer richtigliegen - weder im Alltag noch in der Philosophie. Damit hängt auch Williamsons Replik auf den ersten Einwand zusammen: Dass verschiedene Personen unterschiedliche Reaktionen auf die von Epistemologinnen und Epistemologen angeführten

3 Vgl. Baz 2012, S. 87f., Baz 2016b, S. 111, 114.

4 Vgl. Baz 2012, S. 88f. Als einschlägige Stellen gibt Baz die folgenden an: Williamson 2007, S. 3, 192ff., Williamson 2005, S. 12, Williamson 2004, S. $15^{2}$.

5 Vgl. Baz 2012, S. 91, 95. Vgl. auch Williamson 2007, S. 191 f. 
Fallbeispiele zeigen, liege daran, dass wir nicht alle gleich gut seien in der Klassifikation komplexer Fälle als Fälle von Wissen oder Nicht-Wissen. ${ }^{6}$

Wie stichhaltig diese Argumente von Williamson sind, braucht uns im vorliegenden Kontext nicht zu interessieren, da es noch eine andere Möglichkeit der Erwiderung auf die beiden oben genannten Einwände gibt, die bei Baz ebenfalls anklingt und die der in der vorliegenden Abhandlung verteidigten Philosophie-Konzeption näherliegt: Die Methode möglicher Fälle ist eine Methode zur Analyse unserer Begriffe, beispielsweise unseres Begriffs des Wissens. Wenn wir philosophische Gedankenexperimente daraufhin beurteilen, ob eine ihrer Protagonistinnen über Wissen hinsichtlich einer bestimmten Proposition verfügt, stützen wir uns dabei auf unsere Sprachkompetenz, die wir jeden Tag trainieren ( $a d$ Einwand 2). ${ }^{7}$ Und Unterschiede in der reflektierten Anwendung des Wortes »wissen « zwischen zwei Personen oder Kulturen deuten ganz einfach darauf hin, dass es verschiedene Wissensbegriffe gibt. Da unsere philosophischen Probleme im Kontext unserer eigenen Begriffe aufgetreten sind, interessieren wir uns auch in erster Linie für die Analyse unserer eigenen Begriffe (ad Einwand 1). ${ }^{8}$

Baz zufolge liegen die Verteidigerinnen und Verteidiger der Methode möglicher Fälle falsch in ihrer Annahme einer Kontinuität zwischen alltäglichen Verwendungen des Ausdrucks »wissen« und den Verwendungen, zu denen wir von Epistemologinnen und Epistemologen im Zusammenhang mit philosophischen Gedankenexperimenten aufgefordert werden. ${ }^{9}$ Wir können bei der Beantwortung der Frage des Epistemologen - »Weiß $S$ (in einem bestimmten Gedankenexperiment), dass $p$ ? « nicht auf unsere alltägliche Kompetenz in der Klassifikation von Fällen beziehungsweise unsere im Alltag trainierte Sprachkompetenz zurückgreifen, da die Frage des Epistemologen nicht dasselbe bedeutet wie alltägliche Fragen der Form »Weiß $S$, dass $p$ ?«. Diese Idee veranschaulicht Baz zunächst anhand eines bekannten Gedankenexperiments von Weinberg, Nichols und Stich:

Bob has a friend, Jill, who has driven a Buick for many years. Bob therefore thinks that Jill drives an American car. He is not aware, however, that her Buick has recently been stolen, and he is also not aware that Jill has replaced it with a

6 Vgl. Baz 2012, S. 97, 102. Vgl. auch Williamson 2004, S. 150.

7 Vgl. Baz 2012, S. 96.

8 Vgl. Baz 2012, S. 99. Vgl. auch Grice 1989, S. 175. Baz kritisiert diese Replik auf den Einwand 1 auf der Grundlage von Argumenten, die für das vorliegende Kapitel nicht relevant sind (vgl. Baz 2012, S. 99-101) und mit denen ich mich im Kapitel 7 des vorliegenden Buchs im Zusammenhang mit der Experimentellen Philosophie auseinandersetzen werde.

9 Vgl. Baz 2012, S. 91 . 
Pontiac, which is a different kind of American car. Does Bob really know that Jill drives an American car, or does he only believe it? ${ }^{10}$

Das geschilderte Szenario könnte im Prinzip so im Alltag auftreten. Aber auch wenn es aus bestimmten Perspektiven natürlich wäre, in Bezug auf die geschilderte Situation den Fragesatz »Weiß Bob (wirklich), dass Jill einen amerikanischen Wagen fährt?« zu äußern, so würden diese natürlichen Äußerungen doch nicht die Frage zum Ausdruck bringen, die Epistemologinnen und Epistemologen im Sinn haben. ${ }^{11}$ So jedenfalls Baz' Auffassung, für die er argumentiert, indem er verschiedene Perspektiven durchgeht, aus denen das Szenario betrachtet werden könnte:

\section{Informierter Betrachter}

Für jemanden, der über das Szenario so gut informiert ist, wie wir es als Leserinnen und Leser der obigen Beschreibung sind, stellen sich hinsichtlich der Informiertheit von Bob darüber, dass Jill einen amerikanischen Wagen fährt, keine Fragen. Es gibt keinen Grund, weshalb wir in einer alltäglichen Situation, in der wir als informierte Betrachterinnen oder Betrachter am obigen Szenario beteiligt sind, fragen sollten: »Weiß Bob (wirklich), dass Jill einen amerikanischen Wagen fährt? «, da wir ja schon genau wissen, welche Überzeugung Bob zu diesem Thema hat und worauf sie beruht.12

\section{Agent}

Betrachten wir den Fall stattdessen aus der Perspektive einer weniger gut informierten Person, »Agent«, für die es wichtig ist, zu wissen, ob Jill einen amerikanischen Wagen fährt. Agent trifft Bob, der zu ihr sagt, dass Jill einen amerikanischen Wagen fährt. Sie ist aber nicht sicher, ob sie Bobs Aussage Glauben schenken soll. Also fragt sie sich: »Weiß Bob (wirklich), dass Jill einen amerikanischen Wagen fährt? «. Baz zufolge ist die Frage, die Agent stellt, nicht die Frage des Epistemologen. Denn die Frage des Epistemologen zielt darauf

\footnotetext{
10 Baz 2012, S. 106f. Entnommen aus Weinberg/Nichols/Stich 2001, S. 443. Die Formulierung der Frage zum Schluss der Fallbeschreibung - übersetzt: »Weiß Bob wirklich, dass Jill einen amerikanischen Wagen fährt, oder glaubt er es nur?« - scheint mir ein bisschen unglücklich gewählt zu sein. $\gg S$ glaubt nur, dass $p$ « legt nämlich nahe, dass entweder $p$ nicht wahr ist oder $S$ nicht ganz sicher ist, ob $p$. Besser wäre die Formulierung »Weiß Bob, dass Jill einen amerikanischen Wagen fährt, oder weiß er es nicht?«.

11 Vgl. Baz 2016a, S. 67.

12 Vgl. Baz 2012, S. 108.
} 
ab, ob in einem Fall, der so strukturiert ist wie ein Gettier-Fall, ${ }^{13}$ Wissen vorliegt. Das ist aber nicht das, was Agent wissen will, die ja gar nicht weiß, dass sie es mit einem Gettier-Fall zu tun hat: Sie weiß schließlich nicht, dass Jill tatsächlich einen amerikanischen Wagen fährt, dass aber Bobs wahre, diesbezügliche Überzeugung auf einer falschen Annahme beruht, nämlich auf der Annahme, dass Jill nach wie vor ihren Buick fährt. Was Agent wissen will, so Baz, ist entweder, welche Evidenz Bob dafür hat, dass Jill einen amerikanischen Wagen fährt, oder, falls sie das schon weiß, ob Bobs Evidenz ihr, Agent, einen ausreichend guten Grund dafür gibt, sich darauf zu verlassen, dass Jill einen amerikanischen Wagen fährt. Keine dieser beiden Fragen ist auf das gerichtet, was jemanden, der die Frage des Epistemologen stellt, interessiert, und wenn Agent eine dieser beiden Fragen oder eine damit zusammenhängende Frage mit den Worten »Weiß Bob, dass Jill einen amerikanischen Wagen fährt?« stellt, stellt sie damit eine Frage, die nicht gleichbedeutend mit der Frage des Epistemologen ist. ${ }^{14}$

\section{Judge}

Eine dritte Perspektive, aus der wir den Fall um Bob und Jills Wagen betrachten könnten, ist die Perspektive von »Judge«, der weiß, dass Jills Buick vor Kurzem gestohlen wurde und dass Jill ihn durch einen Pontiac ersetzt hat, und der auch darüber informiert ist, dass Bob annimmt, dass Jill nach wie vor ihren Buick fährt. Judge beobachtet, wie Bob Agent gegenüber behauptet, dass er wisse, dass Jill einen amerikanischen Wagen fährt, und fragt sich, ob es Bob angesichts der Anhaltspunkte, über die er verfügt, zusteht, zu behaupten, dass er weiß, dass Jill einen amerikanischen Wagen fährt. Diese Frage ist Baz zufolge nicht die Frage des Epistemologen: Um die Frage zu beantworten, ob es Bob zusteht, zu behaupten, er wisse, dass Jill einen amerikanischen Wagen fährt, muss Judge, so Baz, davon abstrahieren, dass Jill tatsächlich einen amerikanischen Wagen fährt und dass Bob dies auf der Grundlage einer falschen Überzeugung annimmt: Er muss sich einzig die Frage stellen, ob es aus Bobs Perspektive angemessen ist, zu behaupten, dass er wisse, dass Jill einen amerikanischen Wagen fährt. Und dies ist auch wieder nicht die Frage, ob jemand in einem Gettier-Fall über Wissen verfügt, denn natürlich weiß Bob nicht, dass er sich in einem entsprechend strukturierten Fall befindet. ${ }^{15}$

13 Für Fälle, die so strukturiert sind wie Gettier-Fälle, verwende ich nachfolgend der Einfachheit halber auch dann die Bezeichnung »Gettier-Fall«, wenn sie nicht von Edmund Gettier stammen.

14 Vgl. Baz 2012, S. 108-110.

15 Vgl. Baz 2012, S. 111f. 
Baz bespricht auch noch weitere Perspektiven, auf die es sich im vorliegenden Kapitel nicht einzugehen lohnt, da sie entweder ganz ähnlich geartet sind wie die oben beschriebenen Fälle oder noch weniger aussichtsreiche Kandidaten für Perspektiven darstellen, aus denen sich im Alltag die Frage des Epistemologen stellen könnte. ${ }^{16}$ Contra Baz bin ich allerdings der Meinung, dass sich die Frage des Epistemologen aus bestimmten Perspektiven ganz natürlich ergibt:

\section{Adviser}

Gehen wir wieder von einer Akteurin aus, »Agent ${ }_{2}$ «, für die es wichtig ist, zu wissen, ob Jill einen amerikanischen Wagen fährt oder nicht. Agent ${ }_{2}$ zieht in Erwägung, sich bei Bob zu erkundigen, ob Jill einen amerikanischen Wagen fährt, und sucht Rat bei ihrer Freundin Adviser: »Weiß vielleicht Bob, ob Jill einen amerikanischen Wagen fährt?«. (Aus irgendeinem Grund geht Agent ${ }_{2}$ davon aus, dass ihre Freundin nicht weiß, ob Jill einen amerikanischen Wagen fährt). Adviser ist eine informierte Betrachterin, also so gut über das fragliche Szenario informiert wie wir. Baz kann nun auch hier wieder sagen: Die Frage, die Agent ${ }_{2}$ stellt, ist nicht die Frage des Epistemologen, denn Agent ${ }_{2}$ weiß gar nicht, dass sie es mit einem Gettier-Fall zu tun hat, und die Frage des Epistemologen ist darauf gerichtet, herauszufinden, ob Personen in GettierFällen über Wissen verfügen. Damit hat Baz Recht. Dass Agent ${ }_{2}$ nicht die Frage des Epistemologen stellt, sieht man bereits daran, dass sie fragt: »Weiß Bob, $o b$ Jill einen amerikanischen Wagen fährt?«, und nicht: »Weiß Bob, dass Jill einen amerikanischen Wagen fährt?«. Die »dass «-Frage wird in der Regel von Personen gestellt, die wissen, dass Jill einen amerikanischen Wagen fährt, und dies trifft auf Agent ${ }_{2}$ ja gerade nicht zu. ${ }^{17}$ Der springende Punkt ist jedoch: Wenn Agent $_{2}$ eine informierte Betrachterin wie Adviser fragt, ob Bob weiß, ob Jill einen amerikanischen Wagen fährt, dann stellt sich für Adviser tatsächlich die Frage des Epistemologen: Sie muss sich dann fragen, ob sie über Bob angesichts dessen, dass er in Bezug auf Jills Besitz eines amerikanischen Wagens über eine wahre Überzeugung verfügt, die auf einer falschen Annahme beruht, sagen sollte, dass er wei $\beta$, dass Jill einen amerikanischen Wagen fährt, oder nicht. Natürlich wäre Agent ${ }_{2}$ auch gedient, wenn Adviser sie ganz einfach darüber informiert, dass Jill einen amerikanischen Wagen fährt, oder wenn Adviser ihr versichert, dass Bob ihr jedenfalls diese Frage richtig beantworten wird. Trotzdem haben wir es hier mit einem Kontext zu tun, in dem es für

16 Vgl. Baz 2012, S. 112-115.

17 Vgl. zum dass/ob-Unterschied auch Hanfling 1985, S. 48. 
jemanden natürlich wäre, die oben genannte Frage nach der korrekten Beschreibung von Bob zu stellen, die der Frage des Epistemologen entspricht.

$$
\text { Judge }_{2}
$$

Eine weitere Perspektive, aus der wir den Fall betrachten können, ist die Perspektive von Judge ${ }_{2}$, der wie Judge weiß, dass Jills Buick vor Kurzem gestohlen wurde, dass Jill ihn durch einen Pontiac ersetzt hat, und dass Bob annimmt, Jill fahre immer noch ihren Buick. Wenn Judge ${ }_{2}$ mitbekommt, wie Bob Agent gegenüber behauptet, er wisse, dass Jill einen amerikanischen Wagen fährt, kann er sich die Frage stellen, die Judge ${ }_{(1)}$ sich stellt: Ist es übereilt von Bob, auf der Grundlage der ihm zur Verfügung stehenden Evidenz Wissen für sich zu beanspruchen, oder ist diese Inanspruchnahme von Wissen aus Bobs Perspektive angemessen? Baz hat Recht damit, dass dies nicht die Frage des Epistemologen ist. Judge ${ }_{2}$ kann aber stattdessen auch die Frage stellen: Sagt Bob etwas Wahres, wenn er behauptet, er wisse, dass Jill einen amerikanischen Wagen fährt? Stimmt es, dass er das weiß? Und da Judge ${ }_{2}$ über alle für die Beantwortung dieser Frage relevanten Informationen verfügt, kann diese Frage wieder nur auf die bereits unter »Adviser « formulierte Frage des Epistemologen hinauslaufen: Ist es angesichts der Fakten korrekt, Bob so zu beschreiben, dass er weiß, dass Jill einen amerikanischen Wagen fährt?

\section{Historiker}

Nehmen wir an, ein Historiker schreibt Jahre nachdem Jill ihren Buick durch einen Pontiac ersetzt hat, eine Biografie über Bob und ist an irgendeiner Stelle versucht, zu schreiben, Bob habe zu dem Zeitpunkt, der im Weinberg/Nichols/ Stich-Fall beschrieben wird, gewusst, dass Jill einen amerikanischen Wagen fuhr. Wir können uns leicht eine Voraussetzung vorstellen, unter der es von Interesse wäre, eine entsprechende Information in die Biografie von Bob einzubauen. Zum Beispiel macht Bob in Anwesenheit von Jill eine abwertende Bemerkung über Personen, die amerikanische Wagen fahren, und dies ist einer von mehreren Auslösern dafür, dass seine langjährige Freundschaft mit Jill in die Brüche geht. Der Historiker ist sich nicht ganz sicher, ob es korrekt wäre, zu schreiben, dass Bob diese Bemerkung gemacht habe, obwohl er wusste, dass Jill einen amerikanischen Wagen fuhr, oder ob dies im Lichte dessen, dass Bob irrtümlich davon ausging, dass Jill nach wie vor ihren Buick fuhr, die Sachlage nicht ganz richtig darstellen würde. Diese Überlegung: Wäre es korrekt oder inkorrekt, über Bob zu sagen, er habe gewusst, dass Jill einen amerikanischen Wagen fuhr, drückt wieder exakt die Frage des Epistemologen aus.

Bazgibt die folgende Begründung dafür, dass sich die Frage des Epistemologen im Alltag nicht stellt: »[Q]uestions that arise naturally and intelligibly in the 
course of everyday experience are tied to our practical needs and interests and more broadly to the rest of our practical life, as the theorist's question is not.«18 Mit dem Historiker habe ich einen Fall beschrieben, in dem die Beantwortung der Frage des Epistemologen von einer gewissen praktischen Relevanz ist: Der Historiker schreibt ein Buch und muss sich für eine Formulierung entscheiden. Sollte es korrekt sein, über Bob zu sagen, dass er seine abwertende Bemerkung über die Besitzerinnen und Besitzer amerikanischer Wagen im Wissen gemacht hat, dass Jill einen amerikanischen Wagen fuhr, dann wäre diese Formulierung gegenüber anderen Formulierungen zu bevorzugen: Bob nahm richtigerweise an, dass Jill einen amerikanischen Wagen fuhr « könnte den falschen Eindruck vermitteln, Bob sei sich darüber nicht ganz sicher gewesen. »Bob war sicher, dass Jill einen amerikanischen Wagen fuhr« könnte nahelegen, dass Jill in Wirklichkeit keinen amerikanischen Wagen fuhr. Und den ganzen Sachverhalt präzise zu beschreiben, wäre unnötig umständlich, sofern auch »Bob wusste, ...« korrekt wäre. Contra Baz scheint es also Fälle zu geben, in denen praktische Interessen von der Beantwortung der Frage des Epistemologen abhängen.

Für Adviser und Judge ${ }_{2}$ ist die Beantwortung der Frage des Epistemologen demgegenüber in der Tat nicht von praktischem Interesse. Wie bereits bemerkt, ist es nicht wichtig, dass Adviser die von Agent ${ }_{2}$ gestellte Frage beantwortet. Sie könnte ihr auch einfach die Auskunft geben, die Agent ${ }_{2}$ wirklich benötigt, und ihr mitteilen, dass Jill einen amerikanischen Wagen fährt. Und Judge $_{2}$ stellt die Frage des Epistemologen als nicht involvierter Beobachter und nur aus Interesse. Aber dass wir dies im Alltag regelmäßig machen, Fragen nur aus Interesse stellen, Fragen, die eben nicht mit unseren praktischen Bedürfnissen und Interessen zusammenhängen, sollte auch unabhängig von den oben beschriebenen Szenarien um Adviser und Judge ${ }_{2}$ offensichtlich sein - so offensichtlich, dass anzunehmen ist, dass auch Baz es nicht bestreiten würde. ${ }^{19}$

Wie sich gezeigt hat, können wir uns problemlos Situationen vorstellen, in denen wir im Alltag mit der Frage des Epistemologen konfrontiert werden. Baz kann seine Behauptung, dass wir nicht über die Kompetenz verfügen, die Frage des Epistemologen zu beantworten, also nicht stichhaltig damit begründen, dass die Frage des Epistemologen von einer Art ist, die sich uns im Alltag nie stellen würde.

18 Baz 2016b, S. 12of., Hervorhebung von mir.

19 Vgl. dazu auch Hansen 2018, S. 12f. der PDF-Version. 
Kommen wir nun zu Baz' zweitem Argument gegen die Brauchbarkeit der Methode möglicher Fälle und zur Behauptung, die Frage des Epistemologen habe keine Bedeutung, da ihr der dafür notwendige »Witz« fehle.

Verwendungen von Sätzen der Form $» S$ weiß, dass $p$ « oder von Fragesätzen der Form »Weiß $S$, dass $p$ ? « haben für gewöhnlich einen Witz: Diese Sätze werden mit einer bestimmten Absicht oder aus einem bestimmten (über die Beantwortung der Frage hinausgehenden) Interesse geäußert. ${ }^{20}$ Und abhängig vom je einzelnen Witz haben sie laut Baz unterschiedliche Bedeutungen. ${ }^{21}$ Mit dieser Auffassung wendet sich Baz gegen die ihm zufolge vorherrschende Auffassung des Funktionierens des Wortes »wissen«: Dieses Wort soll eine Relation bezeichnen, die zwischen jeder Person und jeder Tatsache oder wahren Proposition entweder besteht oder nicht besteht. ${ }^{22}$ Und kompetente Sprecherinnen und Sprecher sollen dazu in der Lage sein, in Bezug auf jedes Szenario, über das sie ausreichend informiert sind, zu bestimmen, ob die Personen in dem Szenario hinsichtlich bestimmter Propositionen über Wissen verfügen oder nicht. ${ }^{23}$ Baz zufolge ist ein solches »pure judgment « beziehungsweise eine »sheer application«, ein reines Anwenden eines Ausdrucks auf Fälle, unabhängig von einem bestimmten Witz der Verwendung, im Falle von »wissen (dass $p$ ) « nicht möglich. ${ }^{24}$ Und unsere Kompetenz in der Verwendung des Ausdrucks »wissen « beläuft sich entsprechend auch nicht auf die Fähigkeit, Fälle einzuteilen in Fälle von Wissen und Fälle von Nicht-Wissen. ${ }^{25}$ Stattdessen besteht unsere Kompetenz darin, den Witz von Äußerungen der Form $» S$ weiß, dass $p \ll$ bzw. von Fragen der Form »Weiß $S$, dass $p$ ? « zu erkennen und entsprechend auf sie zu reagieren. ${ }^{26}$

$20 \quad$ Vgl. Baz 2016a, S. 79.

21 Vgl. Baz 2012, S. 117, 121.

22 Man könnte natürlich auch sagen, dass die Relation zwischen jeder Person und jeder Proposition - ob wahr oder falsch - entweder bestehe oder nicht bestehe. Bei falschen Propositionen würde sie dann einfach trivialerweise nie bestehen. Der Unterschied zwischen der obigen Variante und der Variante in der vorliegenden Fußnote spielt für Baz' Argumentation keine Rolle.

23 Vgl. Baz 2012, S. 95.

24 Vgl. Baz 2012, S. 42-45, 94, 119-121, 139.

25 »Fälle einteilen in Fälle von Wissen und Fälle von Nicht-Wissen« ist keine ideale Beschreibung. Die Idee ist, dass man einen Ausdruck wie »wissen, dass es in unserem Sonnensystem acht Planeten gibt « nehmen und dann Personen einteilen kann in solche, auf die dieses Prädikat zutrifft, und solche, auf die es nicht zutrifft. Baz zufolge liegt dieser Idee eine falsche Auffassung des Funktionierens des Ausdrucks »wissen« zugrunde.

Vgl. Baz 2012, S. 105, 120, 189, Baz 2016a, S. 71, Baz 2016b, S. 125. 
Baz gibt diverse Beispiele dafür, welches der Witz des Stellens einer Frage der Form »Weiß $S$, dass $p$ ? « sein kann:

Someone has given you an assurance and you wonder whether it is good enough; someone gave someone else an assurance and you wonder whether it was right for her to do so; $[\ldots]$ you think someone would be interested to learn that such and such, and you wonder whether she already has; someone could have been expected to do something if he had been aware of the fact that such and such, and you wonder whether you are justified in getting angry with him for not having done it; you're simply curious to find out whether someone is aware of the fact that such and such; and so on and so forth. ${ }^{27}$

Je nachdem, aus welchem Interesse eine Frage der Form »Weiß $S$, dass $p$ ? « gestellt wird, sind unterschiedliche Reaktionen angemessen. Wenn ich wissen will, ob meine Kollegin Martha weiß, dass unsere Sitzung verschoben wurde (da ich sie sonst schnell anrufen und ihr Bescheid geben müsste), zielt meine $»$ Weiß $S$, dass $p \ll$-Frage darauf ab, sicherzustellen, dass Martha nicht annimmt, dass die Sitzung zum ursprünglich vereinbarten Termin stattfindet. Auf welcher Grundlage Martha zur Einsicht gelangt ist, dass die Sitzung verschoben wurde, beziehungsweise wie gut ihre Evidenz dafür ist, interessiert mich in einem solchen Fall nicht und es wäre vermutlich unangemessen, mir als Antwort auf meine Frage auseinanderzusetzen, dass Martha sich bezüglich der Verschiebung der Sitzung in einem Gettier-Fall befindet.

Anders verhält es sich, wenn ich, wie Agent im auf S. 115f. beschriebenen Fall, auf der Suche nach einer Person bin, die mir sagen kann, ob Jill einen amerikanischen Wagen fährt, und Bob frage, ob er weiß, ob Jill einen amerikanischen Wagen fährt. Zumindest wenn es für mich besonders wichtig ist, dass ich bezüglich Jills Besitz eines amerikanischen Wagens über eine korrekte Information verfüge, werde ich mich nicht damit zufriedengeben, dass Bob mir versichert, er wisse, dass sie einen amerikanischen Wagen fahre, sondern auch wissen wollen, auf welcher Grundlage sein Wissensanspruch beruht: welche Evidenz er dafür hat, dass Jill einen amerikanischen Wagen fährt.

Baz geht davon aus, dass die Frage »Weiß $S$, dass $p$ ? « je nachdem, aus welchem Interesse sie gestellt wird, nicht nur unterschiedliche Reaktionen angemessen macht, sondern auch unterschiedliche Bedeutungen hat. Diese Idee findet sich auch bei anderen Philosophinnen und Philosophen, die andere Konsequenzen daraus ziehen als Baz. Einer dieser Philosophen ist Gerhard Ernst. Dessen Position zu den unterschiedlichen Bedeutungen des Ausdrucks 
»wissen« soll nachfolgend zur Abgrenzung von Baz’ Position kurz dargelegt werden.

Ernst zufolge hat der Satz $\ S$ weiß, dass $p$ « unterschiedliche Bedeutungen, je nachdem, ob er aus der Perspektive einer Person geäußert wird, die weiß, dass $p$, oder aus der Perspektive einer Person, die nicht weiß, dass $p .{ }^{28}$ Und dasselbe gilt für den entsprechenden Fragesatz. Wenn ich weiß, dass $p$, und in Bezug auf eine andere Person frage: $»$ Weiß $S$, dass $p$ ? «, dann mache ich das typischerweise deshalb, weil ich $S$, sofern er oder sie noch nicht darüber informiert ist, mitteilen möchte, dass $p$, oder weil es für meine Bewertung einer Handlung von $S$ relevant ist, ob er oder sie zum Zeitpunkt der Ausführung der Handlung wusste, dass $p$. Ich interessiere mich als Wissender oder Wissende für andere Personen also als potentielle Informations-Empfängerinnen und -Empfänger oder als Akteurinnen und Akteure. Die entsprechenden Interessen nennt Ernst die »Interessen des Wissenden«. Wenn ich im oben beschriebenen Beispiel frage, ob Martha weiß, dass die Sitzung verschoben wurde, ist meine Frage gleichbedeutend mit der Frage, ob Martha sich der Tatsache bewusst ist, dass die Sitzung verschoben wurde - und diese Frage läuft Ernst zufolge auf die Frage hinaus, ob Martha diesbezüglich eine wahre Überzeugung hat. ${ }^{29}$

Wenn ich nicht weiß, dass $p$, und die Frage »Weiß $S$, ob $p$ ? « folglich mit den »Interessen des Unwissenden« stelle, zielt sie, so Ernst, darauf ab, einen Informanten oder eine Informantin zu finden. Die Frage, die Adviser im Beispiel auf S. 117 gestellt wird, lässt sich mithin nicht adäquat paraphrasieren mit »Hat Bob eine wahre Überzeugung in Bezug auf Jills Besitz eines amerikanischen Wagens? «, sondern bedeutet: »Ist Bob in Bezug auf Jills Besitz eines amerikanischen Wagens ein guter Informant? «.Gute Informantinnen und Informanten in Bezug auf $p$ haben nicht nur die wahre Überzeugung, dass $p$, sondern können auch sämtliche Zweifel, die ihr Gegenüber in Bezug auf $p$ hat, aus dem Weg räumen beziehungsweise alle Alternativen zu $p$, die ihr Gegenüber in Betracht zieht, ausschließen. ${ }^{30}$ Ernst nimmt auf wahre Überzeugungen auch als »objektive Variante von Wissen« Bezug, und nennt das, worüber gute

28 Vgl. Ernst 2002, Ernst 2012. Eine Person, die nicht weiß, dass $p$, würde natürlich nicht den Satz $S$ weiß, dass $p$ « äußern, ebenso wenig wie sie im Normalfall fragen würde: »Weiß $S$, dass $p$ ? «. Stattdessen würde sie, wie bereits weiter oben erwähnt, fragen: »Weiß $S$, ob $p$ ? «. Diesen Punkt können wir jedoch hier ausklammern, da es Ernst um eine Perspektive des Unwissenden geht, die wir zu theoretischen Zwecken auch dann einnehmen können, wenn wir selbst über das betreffende Wissen verfügen, so dass die »dass«-Formulierung adäquat ist.

29 Vgl. Ernst 2012, S. 315. »Überzeugung « wird dabei von Ernst in einem etwas anspruchsvolleren Sinn verstanden, der unter anderem einen bestimmten Grad von Rationalität beim betreffenden Subjekt voraussetzt (vgl. Ernst 2012, S. 315f.).

Vgl. Ernst 2012, S. 310. 
Informantinnen und Informanten verfügen, "perspektivische Variante von Wissen«. Dies deshalb, weil der oben definierte Begriff des guten Informanten oder der guten Informantin relational ist: Da verschiedene Akteurinnen und Akteure unterschiedliche Zweifel an $p$ haben können beziehungsweise unterschiedliche Alternativen zu $p$ in Betracht ziehen können, lässt sich die Frage, ob jemand ein guter Informant oder eine gute Informantin ist, immer nur bezogen auf die Perspektive eines bestimmten Akteurs oder einer bestimmten Akteurin beantworten. ${ }^{31}$

Dass eine Frage der Form »Weiß $S$, dass $p$ ? « nicht nur abhängig davon, ob die objektive oder die perspektivische Variante von Wissen gemeint ist, unterschiedliche Antworten haben kann, sondern im Falle der perspektivischen Variante auch abhängig davon, von welchem Standpunkt aus sie zu beantworten ist, liefert Ernst zufolge eine Teilerklärung für die Unentschlossenheit, die uns manchmal bei der Beurteilung von epistemologischen Gedankenexperimenten beziehungsweise bei der Beantwortung der entsprechenden Fragen des Epistemologen erfasst:Je nach Blickwinkel tendieren wir zu der einen oder der anderen Antwort, sind uns aber nicht dessen bewusst, dass die Bedeutung der Frage des Epistemologen nicht eindeutig bestimmt ist. ${ }^{32}$

Wenn man der Auffassung ist, dass Fragen der Form »Weiß $S$, dass $p$ ? situationsabhängig (bzw. abhängig vom Interesse, aus dem sie gestellt werden) Unterschiedliches bedeuten können, scheint es nur konsequent, mit Ernst zu behaupten, die Frage des Epistemologen sei mehrdeutig. ${ }^{33}$ Wie kommt es, dass Baz, der ebenso wie Ernst glaubt, dass Fragen der Form »Weiß $S$, dass $p$ ? « je nachdem, aus welchem Interesse sie gestellt werden, Unterschiedliches bedeuten können, stattdessen zum Schluss kommt, die Frage des Epistemologen habe keine Bedeutung? Hierzu sind zwei Dinge zu sagen: Erstens hat es den Anschein, dass Baz noch eine viel größere Variabilität der Bedeutung von $» S$ weiß, dass $p$ « beziehungsweise »Weiß $S$, dass $p$ ? « zulassen möchte als Ernst.

$31 \quad$ Vgl. Ernst 2012, S. 311.

32 Vgl. Ernst 2012, S. 325, Ernst 2015, S. 18 f.

33 Ernsts Idee ist eigentlich etwas differenzierter: Er schreibt zwar an verschiedener Stelle explizit, der Satz $\gg S$ weiß, dass $p$ « bedeute in verschiedenen Äußerungskontexten Unterschiedliches, möchte in diesem Zusammenhang aber ungern von einer »Ambiguität« sprechen: Da das Phänomen, dass Ausdrücke, je nach Kontext, nicht exakt dasselbe bedeuten, in natürlichen Sprachen derart oft auftritt, würden sich andernfalls, so Ernst, zu viele Ausdrücke als ambig herausstellen. Er entscheidet sich stattdessen, von »Bedeutungsvarianten« zu sprechen (vgl. Ernst 2012, S. 307). Andernorts räumt Ernst demgegenüber ein, dass der Ausdruck »wissen« mehrdeutig ist, wenn auch in weniger »radikaler« Weise als beispielsweise »Bank« oder »Schimmel«. Im Gegensatz zu Letzteren drückt »wissen« Ernst zufolge nicht unterschiedliche Begriffe aus, sondern verschiedene Varianten desselben Begriffs (vgl. Ernst 2002, S. 48f.). 
An einigen Stellen scheint Baz nahezulegen, dass Äußerungen dieser Sätze je nach Kontext fast Beliebiges bedeuten können. Wenn dies der Fall ist, lässt sich die Frage des Epistemologen nicht disambiguieren und für jede der verschiedenen Lesarten separat beantworten wie bei Ernst, sondern es wäre unklar, wie man mit einer Disambiguierung überhaupt beginnen könnte. Ich werde im Abschnitt 6 des vorliegenden Kapitels die Passagen in When Words Are Called For diskutieren, die auf eine solche extreme Variabilität in der Bedeutung von $» S$ weiß, dass $p$ « hindeuten, und in diesem Kontext auch noch einmal auf Ernsts Auffassung zurückkommen.

Die zweite und wichtigere Erklärung dafür, dass die Frage des Epistemologen Baz zufolge nicht mehrdeutig ist, sondern nichts bedeutet, ist die folgende: Es ist nicht der Fall, dass wir beispielsweise bezogen auf das obige Gedankenexperiment nicht wissen, aus welchem Interesse Weinberg, Nichols und Stich (nachfolgend auch: »WNS «) die Frage »Weiß Bob, dass Jill einen amerikanischen Wagen fährt? « stellt oder, um Ernsts Unterscheidung aufzugreifen, ob sie wissen wollen, ob Bob diesbezüglich über eine wahre Überzeugung verfügt, oder wissen wollen, ob Bob ein guter Informant wäre. WNS sind so gut über Bobs Verhältnis zum Umstand, dass Jill einen amerikanischen Wagen fährt, informiert, wie es nur geht (denn sie haben den Fall selbst kreiert, und es kann keine Aspekte des Falls geben, von denen die drei keine Kenntnis haben). Sie wissen, dass Jill einen amerikanischen Wagen fährt, und sind also nicht auf der Suche nach einem Informanten. Und sie wissen, dass Bob eine wahre Überzeugung in Bezug auf Jills Besitz eines amerikanischen Wagens hat, und stellen die Frage also nicht mit dem Interesse von jemandem, der Bob gegebenenfalls über diesen Umstand informieren möchte. Ebenso wenig stellen WNS ihre Frage mit dem Interesse von jemandem, der damit befasst ist, irgendwelche Handlungen von Bob zu bewerten. Das Interesse, mit dem WNS die Frage des Epistemologen stellen, besteht darin, die notwendigen und hinreichenden Bedingungen für das Vorliegen von Wissen zu bestimmen beziehungsweise eine bestimmte Wissenstheorie zu überprüfen, ${ }^{34}$ und dieses Interesse entspricht weder den von Ernst spezifizierten Interessen des Wissenden noch den Interessen des Unwissenden. Folglich, so könnte Baz bezugnehmend auf Ernsts Auffassung argumentieren, kann weder die objektive noch die perspektivische Variante von Wissen gemeint sein, und die Frage des Epistemologen hat nicht zwei oder mehrere mögliche Bedeutungen, sondern überhaupt keine Bedeutung.

Baz schreibt: »There is [...] an important sense in which neither the words expressing the theorist's question nor the words expressing answers to it are 
being used.«35 Dass ein Ausdruck verwendet (»used«) wird, ist dabei in Abgrenzung dazu gemeint, dass er »untätig« (»idle«) ist, nicht dafür eingesetzt wird, eine »echte Arbeit zu verrichten «. ${ }^{36}$ Unsere gewöhnliche Verwendung von »wissen « legt (mit einer bestimmten Flexibilität) fest, welche Arbeiten damit verrichtet werden können. ${ }^{37}$ Mit Ernst könnte man wieder sagen, dass wir damit die oben genannten Interessen des Wissenden oder des Unwissenden verfolgen können und uns beispielsweise danach erkundigen oder darüber austauschen können, ob jemand ein guter Informant oder eine gute Informantin ist. Herauszufinden, welches unser Wissensbegriff ist, gehört nicht zu den Arbeiten, die man mit dem Wort »wissen « verrichten kann, und deshalb wird das Wort »wissen « in der Frage des Epistemologen Baz zufolge auch nicht gebraucht. Die Frage des Epistemologen hat, so Baz, keinen Witz »im relevanten Sinn «. ${ }^{38}$ Wir können zwar die Wörter verstehen, die in der Frage vorkommen, und auch die Frage in dem Sinn verstehen, dass wir Situationen beschreiben können, in denen sie sinnvoll verwendet werden könnte, aber im Kontext des philosophischen Gedankenexperiments bedeutet sie nichts:

As long as we are unable to see the point of an utterance - that is, the speaker's intention as embodied in her speech act - we may still know the meaning of each of her words, in the sense of being their competent employers, but we will not know what each of her words means there and then. ${ }^{39}$

Dieses Problems sind wir uns allerdings üblicherweise nicht bewusst, wenn wir mit der Frage des Epistemologen konfrontiert werden. Da wir die Ausdrücke, die in dieser Frage vorkommen, alle verstehen und vielleicht auch der auf S. 120 erwähnten, vorherrschenden Auffassung des Funktionierens des Wortes »wissen « verhaftet sind, haben wir in Bezug auf die Frage des Epistemologen »Bedeutungs-Halluzinationen«, wie Baz es ausdrückt. ${ }^{40}$

Dass die Frage des Epistemologen in Wirklichkeit nichts bedeutet, erklärt Baz zufolge den Umstand, dass verschiedene Personen, die im Alltag das Wort »wissen« auf dieselbe Weise verwenden, in Bezug auf epistemologische

35 Baz 2016a, S. 79. Vgl. auch Baz 2012, S. 104.

36 Vgl. Baz 2012, S. 2.

37 Vgl. Baz 2012, S. 139, 2016a, S. 69. Bei Baz ist in diesem Zusammenhang immer eher von der Geschichte eines Wortes oder seiner Verwendung die Rede als von seinem gewöhnlichen Gebrauch (vgl. auch Baz 2012, S. 134). Über diesen Unterschied kann aber im vorliegenden Kontext hinweggesehen werden.

38 Vgl. Baz 2012, S. 105.

39 Baz 2016a, S. 71.

40 Vgl. Baz 2012, S. 73. 
Gedankenexperimente oft zu unterschiedlichen Urteilen gelangen. ${ }^{41}$ Und auch dass wir bei der Beantwortung der Frage des Epistemologen den Eindruck haben, uns mit »Intuitionen « behelfen zu müssen, erklärt Baz bezugnehmend auf das von ihm identifizierte Problem mit der Methode möglicher Fälle:

[T] he talk of philosophical intuition [...] registers a sense of lacking sufficient orientation in the theorist's peculiar context - of standing nowhere in particular and having nowhere in particular to go to with one's words. ${ }^{42}$

Aus den vorangehenden Erwägungen sollte bereits hervorgegangen sein, dass es für Baz keinen Ausweg darstellt, einfach zu sagen, dass wir uns für die Beantwortung der Frage des Epistemologen vorstellen müssen, dass sie aus einer anderen Perspektive als derjenigen der Autorin oder des Autors des betreffenden Gedankenexperiments und mit einem anderen Interesse als demjenigen der Bestimmung unseres Wissensbegriffs gestellt wird. Erstens gibt es, wie oben angedeutet, unbestimmt viele Perspektiven, aus denen wir Gedankenexperimente betrachten könnten, und einer bestimmten Lesart von Baz zufolge auch unbestimmt viele Bedeutungen von »Weiß Bob, dass Jill einen amerikanischen Wagen fährt? «, mit jeweils unterschiedlichen korrekten Antworten. Zweitens beschreibt Baz, wie im Abschnitt 2 des vorliegenden Kapitels dargelegt, bereits diverse naheliegende Perspektiven, aus denen man die Frage »Weiß Bob, dass Jill einen amerikanischen Wagen fährt?« stellen könnte, und versucht zu zeigen, dass keine davon auf eine Frage hinausläuft, die Weinberg, Nichols und Stich interessiert. Ob es aus Bobs Perspektive beispielsweise angesichts seiner Anhaltspunkte angemessen ist, zu behaupten, er wisse, dass Jill einen amerikanischen Wagen fahre, ist nicht das, was WNS wissen wollen.

\section{4}

\section{Beschreibungsfragen}

Baz hat Recht damit, dass Weinberg, Nichols und Stich über den Fall um Bob und Jills Wagen bereits so gut informiert sind, wie man nur darüber informiert sein kann, und dass ihre Frage also nicht darauf gerichtet sein kann, eine zusätzliche Information über diesen Fall zu erlangen. In diesem Zusammenhang schreibt Baz auch Folgendes:

41 Vgl. Baz 2012, S. 118. Siehe auch Baz 2017, S. 57 f.

42 Baz 2016a, S. 8o. Vgl. auch Baz 2012, S. 118, Baz 2016b, S. 127. 
Normally, if you say of someone (a politician, for example) that he knows something and I say he does not, what we disagree about are the facts or their significance, not the meaning of 'know(s) that'. We disagree about the case, but we are still in agreement in our use of 'know that' and cognates, and in our understanding of these words. In fact, it is precisely this underlying agreement that makes it possible for us to disagree on particular cases and to go about trying to settle our disagreements. This is precisely what does not happen when, in the context of theorizing about knowledge, you say in the face of some example 'knows' and I say 'does not know' [...], where nothing but a philosophical theory of knowledge hangs on our answers. If the example is to do its intended theoretical work, there should be no disagreement among the respondents about the facts - we all are supposed to know all that any normal person would know about the case, once she has read its author's description of it. If there is genuine disagreement between us here, it seems that it would have to be about the meaning of our words. $^{43}$

Baz formuliert hier eine Einsicht, die eigentlich von vornherein selbstverständlich sein sollte: Natürlich geht es Epistemologinnen und Epistemologen um die Bedeutung des Ausdrucks »wissen«, und nicht darum, »Fakten-Wissen « über die Protagonistinnen und Protagonisten von Gedankenexperimenten zu erlangen. Wie ich im Abschnitt 2 des vorliegenden Kapitels zu zeigen versucht habe, lassen sich aber auch Situationen beschreiben, in denen wir im Alltag Fragen der Form »Weiß $S$, dass $p$ ? « stellen würden, ohne uns damit für FaktenWissen $\mathrm{zu}$ interessieren. Und solche Fragen, diejenigen beispielsweise von Adviser, Judge $_{2}$ und des Historikers, scheinen Sinn zu ergeben - auch wenn Baz darin zuzustimmen ist, dass es nicht einer gewöhnlichen Funktion des Ausdrucks »wissen « oder »Weiß $S$, dass $p$ ? « entspricht, Erkundungen darüber anzustellen, was »wissen« bedeutet. Die Frage des Epistemologen ist ebenso wie die Fragen von Adviser und Judge ${ }_{2}$ und die Frage des Historikers eine $B e$ schreibungsfrage, die darauf gerichtet ist, zu erfahren, ob es korrekt wäre, Bob so zu beschreiben, dass er weiß, dass Jill einen amerikanischen Wagen fährt. Tatsächlich geht Baz nicht völlig über solche Beschreibungsfragen hinweg. Er schreibt in diesem Kontext Folgendes:

The closest that we come in ordinary, 'nonphilosophical' discourse to being in the theorist's peculiar context and attending to something resembling her question is in situations in which disputes arise concerning what we ought to call some given case, or how we ought to describe it, where it is assumed that there is no real disagreement between the parties about the nature of the case itself. But even in those situations, a particular context of significant application is normally in place, or at least assumed or imagined. Indeed, if my argument in the previous section and in the following chapters is on the right track, then, at 
least in the case of 'know that' and its cognates, no determinate question may be raised about a case by means of those words apart from some such context. And this means that the question asked in those ordinary contexts is not the theorist's question of whether the case is a case of $x$ simpliciter. Rather, it is the question of whether we should call it ' $x$ ' or describe it as $x$ given some actual or imagined constellation of interests ('intents and purposes'). Though the focus in such contexts may be on the word, the question is still a question about its (proper) use; and the actual or imagined significance of the case must still be taken into consideration, if only implicitly, in determining what we should call it or how we should describe it. ${ }^{44}$

Baz meint, dass sich auch alltägliche Beschreibungsfragen wesentlich von der Frage des Epistemologen unterscheiden, und zwar insofern, als die Frage des Epistemologen in Bezug auf Fälle gestellt wird, mit denen sich Epistemologinnen und Epistemologen einzig aus dem Grund auseinandersetzen, dass sie herausfinden wollen, welches die notwendigen und hinreichenden Bedingungen für das Vorliegen von Wissen sind. Demgegenüber, so Baz, stellen wir Beschreibungsfragen im Alltag in Bezug auf Fälle, für die wir uns aus irgendwelchen nicht-philosophischen Gründen interessieren. Das Interesse, das wir in solchen Kontexten an der Beantwortung von Beschreibungsfragen haben, geht über das Interesse, aus dem die Frage des Epistemologen gestellt wird, hinaus und hat einen Einfluss auf die Bedeutung von Beschreibungsfragen und mithin darauf, welches ihre korrekte Antwort ist.

Hier kann ich Baz nicht folgen. Welche über die Beschreibungsfrage hinausgehenden Interessen sollten Adviser oder Judge ${ }_{2}$ in den im Abschnitt 2 des vorliegenden Kapitels beschriebenen Fällen haben? Wenn wir den oben beschriebenen Fall des Historikers betrachten, hat dieser an der Frage, ob Bob wusste, dass Jill einen amerikanischen Wagen fuhr, zwar in der Tat ein weitergehendes Interesse: Er stellt diese Frage im Kontext von Bobs Verantwortlichkeit für die Verstimmung von Jill und ihren Kontaktabbruch. Aber dennoch läuft die Frage des Historikers nicht auf die Frage hinaus, ob Bob für Jills Verstimmung und den Kontaktabbruch verantwortlich ist, und beide Fragen sind unabhängig voneinander zu beantworten: Da Bob seine abwertende Bemerkung über Personen mit amerikanischen Wagen in der Überzeugung gemacht hat, dass Jill einen amerikanischen Wagen fuhr, hat er Jills Verstimmung offensichtlich in Kauf genommen und kann dafür kritisiert werden. Aber dies gibt uns keinen Anhaltspunkt dafür, ob die Beschreibung »Bob weiß, dass Jill einen amerikanischen Wagen fährt« korrekt wäre oder nicht. 
Baz bleibt uns eine Erklärung schuldig, wie die nicht-philosophischen Interessen, über die wir im Zusammenhang mit alltäglichen Beschreibungsfragen verfügen, deren Bedeutung beeinflussen sollten, und ich sehe nicht, wo eine solche Erklärung ansetzen könnte. Der hier vertretenen Auffassung nach haben alltägliche Beschreibungsfragen exakt dieselbe Bedeutung wie die Frage des Epistemologen.

Im Zuge seiner Argumentation dafür, dass die Frage des Epistemologen keinen Witz hat und dass nicht klar ist, was das Wort »wissen « im Zusammenhang mit der Frage des Epistemologen leisten soll, macht Baz darauf aufmerksam, dass den Akteuren in Gedankenexperimenten oft unnatürliche Verwendungen des Ausdrucks »wissen« in den Mund gelegt werden. In diesem Punkt stimme ich $\mathrm{Baz} \mathrm{zu}$, und seine Besprechung von Beispielen für dieses Phänomen enthält einige interessante Hinweise auf unseren Gebrauch von »wissen«.

Betrachten wir den folgenden, von Stewart Cohen stammenden Fall:

\begin{abstract}
Mary and John are at the L.A. airport contemplating taking a certain flight to New York. They want to know whether the flight has a layover in Chicago. They overhear someone ask a passenger Smith if he knows whether the flight stops in Chicago. Smith looks at the flight itinerary he got from the travel agent and responds, "Yes I know - it does stop in Chicago." It turns out that Mary and John have a very important business contact they have to make at the Chicago airport. Mary says, "How reliable is that itinerary? It could contain a misprint. They could have changed the schedule at the last minute." Mary and John agree that Smith doesn't really know that the plane will stop in Chicago. They decide to check with the airline agent. ${ }^{45}$
\end{abstract}

Mary äußert Zweifel an der Zuverlässigkeit des Flugplans, den der Passagier Smith konsultiert hat, und dann heißt es: »Mary and John agree that Smith doesn't really know that the plane will stop in Chicago. «Baz macht geltend, dass sich die Diskussion von Mary und John eigentlich nur darum drehen kann, ob sie sich angesichts der für sie verfügbaren Evidenz (nämlich der Angabe in Smiths Flugplan) darauf verlassen wollen, dass der Flug einen Zwischenaufenthalt in Chicago hat, oder ob sie dazu noch weitere Erkundungen durchführen wollen. Die Frage, ob Smith wei $\beta$, dass der Flug einen solchen Zwischenaufenthalt hat, ist für ihr Anliegen unerheblich, und sollten sie zum Schluss kommen, dass sie sich nicht auf Smiths Fahrplan verlassen wollen, hat dies nichts damit

Cohen 1999, S. $5^{8 .}$ 
zu tun, sich »darauf zu einigen, dass Smith nicht wirklich weiß, dass der Flug in Chicago zwischenlandet $\ll .46$

Ähnlich verhält es sich Baz zufolge in anderen Fällen. So zum Beispiel im berühmten Bank-Beispiel von Keith DeRose. Für die Zwecke des vorliegenden Kapitels, reicht es, dieses Beispiel wie folgt zu skizzieren: DeRose sitzt mit seiner Frau am Freitagnachmittag im Auto vor der Bank, in der die beiden ihre Gehaltsschecks einlösen wollen. Sie sehen, dass viele Leute anstehen, und überlegen sich deshalb, ob sie das Unterfangen auf den nächsten Tag verschieben könnten. DeRoses Frau fragt: »Weißt du, ob die Bank morgen geöffnet ist?«, und DeRose antwortet: »Ja, ich weiß, dass sie geöffnet ist, ich war vorletzte Woche am Samstag da.«Da es sehr wichtig ist, dass DeRose und seine Frau ihre Schecks noch diese Woche einlösen, hakt DeRoses Frau noch einmal nach: »Aber Banken ändern manchmal ihre Öffnungszeiten. Weißt du wirklich, dass die Bank morgen geöffnet ist? «. Daraufhin räumt DeRose ein: »Nein, eigentlich weiß ich es nicht. Gehen wir besser hinein und fragen nach. « ${ }^{47}$

Baz macht darauf aufmerksam, dass die Rückfrage von DeRoses Frau, »Weißt du wirklich, dass die Bank morgen geöffnet ist?«, seltsam anmutet. Baz zufolge liegt dies daran, dass DeRose bereits erklärt hat, worauf seine Annahme, dass die Bank am nächsten Tag geöffnet ist, beruht. Wenn ich auf der Suche nach einer Person bin, die mir sagen kann, ob $p$, dann ist es sinnvoll, andere Leute zu fragen: »Weiß irgendjemand, ob $p$ ? « beziehungsweise »Weißt du, ob $p$ ? und mich, falls dies jemand bejaht und die Angelegenheit besonders wichtig für mich ist, vielleicht auch zu erkundigen: »Woher weißt du es? «. Wenn ich aber genau weiß, auf welcher Grundlage jemand behauptet, dass $p$, dann ergibt es keinen Sinn, nachzuhaken: »Aber weißt du (wirklich), dass $p$ ? «. Erstens kann die andere Person in einer solchen Situation nicht besser als ich selbst beurteilen, ob sie über Wissen verfügt, und zweitens habe ich die Frage »Weißt $\mathrm{du}, \mathrm{ob} p$ ? « ja ursprünglich deshalb gestellt, weil ich herausfinden wollte, ob $p$. Ich hätte die andere Person ebenso gut fragen können: »p?«. Sobald sie mich über die Anhaltspunkte für $p$ informiert hat, über die sie verfügt, gibt es für

46 Vgl. Baz 2012, S. 173f. Ein interessanter Hinweis von Baz, der hier nicht unterschlagen werden soll, ist, dass Mary und John einen allfälligen Disput hinsichtlich der Frage, die sie beantworten wollen, tatsächlich unter Einbezug des Wortes »wissen« führen könnten: »Komm, lass uns einfach einchecken, wir wissen ja jetzt dank Smith, dass der Flug einen Zwischenaufenthalt in Chicago hat! « - Nein, wir wissen es eben nicht!«. In einer solchen Debatte würde es John und Mary nach wie vor darum gehen, ob es vernünftig wäre, sich auf den Hinweis von Smith zu verlassen, und nicht um die semantische Frage des Epistemologen, welche Beschreibung von ihnen korrekt wäre (vgl. Baz 2012, S. 176).

Vgl. Baz 2012, S. 165f., DeRose 1992, S. 913. 
mich keinen Grund, mich weiter für das Verhältnis, in dem die andere Person zu $p$ steht, zu interessieren. ${ }^{48}$

Für DeRose und seine Frau stellt sich, nachdem sie geklärt haben, welchen Anhaltspunkt sie dafür haben, dass die Bank am nächsten Tag geöffnet ist, nur die Frage, ob sie nach Hause fahren und sich darauf verlassen wollen, dass die Bank am nächsten Tag geöffnet ist, oder ob sie lieber aussteigen und sich versichern wollen, dass die Bank am Samstag geöffnet ist. Auf die Frage von DeRoses Frau, ob DeRose wirklich wisse, dass die Bank am nächsten Tag geöffnet ist, kann man sich in diesem Kontext keinen Reim machen.

Baz hat Recht damit, dass sich die Fragen, ob Smith weiß, ob der Flug einen Zwischenaufenthalt in Chicago hat, oder ob DeRose weiß, dass die Bank am nächsten Tag geöffnet ist, aus den in den entsprechenden Gedankenexperimenten beschriebenen Perspektiven nicht stellt. Dies heißt jedoch nicht, dass wir als Philosophinnen und Philosophen - und als externe Betrachterinnen und Betrachter der Gedankenexperimente von Cohen und DeRose - die entsprechenden Fragen nicht sinnvoll stellen können. ${ }^{49}$ Die Frage, ob Smith weiß, dass der Flug einen Zwischenaufenthalt in Chicago hat, würde aus dem Mund eines Epistemologen oder einer Epistemologin auf die folgende Frage hinauslaufen: Ist die Evidenz, dass auf dem Flugplan steht, dass der Flug einen Zwischenaufenthalt in Chicago hat, gut genug für Wissen? ${ }^{50}$ Und wollen wir folglich, wenn Smith eine entsprechende Überzeugung auf der Basis des Flugplans gebildet hat und der Flug tatsächlich einen Zwischenaufenthalt in Chicago hat (und kein Gettier-Fall vorliegt), sagen, dass Smith wei $\beta$, dass der Flug einen Zwischenaufenthalt in Chicago hat?

Und analog für den Bank-Fall: Ist der Umstand, dass DeRose vor zwei Wochen am Samstag in der Bank war, ein Anhaltspunkt, der gut genug dafür ist, zu wissen, dass die Bank diesen Samstag wieder geöffnet ist? Wäre es also, wenn DeRose auf der Grundlage dieses Umstands eine entsprechende Überzeugung bildet und die Bank diesen Samstag tatsächlich geöffnet ist (und kein Gettier-Fall vorliegt), korrekt, über ihn zu sagen, er wisse, dass die Bank diesen Samstag geöffnet ist?51

48 Vgl. Baz 2012, S. 167f.

49 Auch im Kontext der Fälle von Cohen und DeRose könnte man natürlich wieder Szenarien wie diejenigen um Adviser, Judge ${ }_{2}$ und den Historiker aus dem Abschnitt 2 des vorliegenden Kapitels konstruieren, in denen sich die Frage, ob Smith respektive DeRose über Wissen verfügen, im Alltag stellen könnte.

5o Wahrscheinlich müsste man noch spezifizieren, wie alt der Flugplan ist.

$5^{1}$ Ich behaupte nicht, dass unser gewöhnliche Gebrauch von »wissen« eine korrekte Antwort auf diese Fragen festlegt, und auch nicht, dass das Stellen dieser Fragen ein 
Baz zufolge beeinflusst der Witz der Äußerung eines Satzes der Form »S weiß, dass $p$ « oder »Weiß $S$, dass $p$ ? « dessen Bedeutung. In When Words Are Called For werden zwar viele Beispiele dafür angeführt, worin ein solcher Witz bestehen kann, ${ }^{52}$ aber Baz äußert sich an keiner Stelle deutlich dazu, wie wir uns die Beeinflussung der Bedeutung von Äußerungen der Form »S weiß, dass $p$ « beziehungsweise »Weiß $S$, dass $p$ ? « durch einen spezifischen Witz vorzustellen haben. Angesichts dessen, wie er bestimmte Beispiele kommentiert, macht es den Eindruck, als schwebe Baz eine äußerst umfassende Beeinflussung vor. ${ }^{53}$ Man betrachte in diesem Zusammenhang etwa seine Besprechung des folgenden Szenarios aus der Feder von Charles Travis:

Hugo, engrossed in the paper, says, 'I need some milk for my coffee.' Odile replies, 'You know where the milk is.' Suddenly defensive, Hugo replies: 'Well, I don't really know that, do I? Perhaps the cat broke into the refrigerator, or there was just now a very stealthy milk thief, or it evaporated or suddenly congealed. ${ }^{54}$

Zu diesem Beispiel schreibt Baz unter anderem:

The most natural way of imagining Travis's example, it seems to me, is to hear Odile as rebuking Hugo for his laziness, or chauvinism, or both. If this is how we hear her words, then it matters very little that she chose words of the form ' $\mathrm{N}$ know(s) that such and such' to make her point. What she says to him is, in effect, that if he wants milk, he should get some himself.55

Baz hat Recht damit, dass Odile Hugo mit ihrer Äußerung zu verstehen gibt, dass er die Milch selbst holen soll. Aber wollen wir wirklich sagen, dass sie ihm dies sagt? Auf den ersten Blick entspricht der Fall einem typischen Beispiel für eine Grice'sche konversationelle Implikatur: Odile verletzt die Konversationsmaxime der Relevanz, indem sie zu Hugo auf seine implizite Aufforderung hin, ihm die Milch zu bringen, etwas sagt, das für die Angelegenheit zunächst einmal nicht von Belang ist, nämlich dass er wisse, wo die Milch ist. Dass Hugo weiß, wo die Milch ist, wäre relevant, wenn nicht Odile, sondern er sie holen würde. Deshalb muss Hugo davon ausgehen, dass Odile ihm genau dies

geeigneter Ansatzpunkt für eine Analyse unseres Wissensbegriffs darstellt. Das einzige, worauf ich bestehen möchte, ist, dass die Fragen sinnvoll sind.

$5^{2}$ Vgl. Zitat auf S. 121 des vorliegenden Kapitels.

53 Vgl. dazu auch Baz 2017, S. 169f. und insbesondere S. 172.

54 Baz 2012, S. 147, entnommen aus Travis 1989, S. 156.

55 Baz 2012, S. 148f. 
nahelegen möchte: Odile sagt, dass Hugo weiß, wo die Milch ist, und implikiert, dass er die Milch selbst holen soll.

Baz hat prinzipiell nichts dagegen, zu unterscheiden zwischen dem, was jemand mit der Äußerung eines Satzes sagt, und dem, was er seiner Zuhörerschaft zusätzlich oder in Abweichung vom Gesagten vermittelt. ${ }^{56}$ Was er ablehnt, ist die Annahme, dass das, was mit der Äußerung eines Satzes wie »Hugo weiß, wo die Milch ist « gesagt wird, durch die Standard-Bedeutung der Wörter festgelegt ist, aus denen der Satz besteht. Baz zufolge müssen wir die kommunikativen Interessen von Odile bereits berücksichtigen, wenn wir bestimmen wollen, was sie mit ihrer Äußerung sagt, und nicht erst, wenn wir uns fragen, ob sie damit ihrem Gegenüber etwas Zusätzliches zu verstehen gibt. Und auf der Grundlage der Betrachtung der spezifischen Interessen von Odile kommt Baz zum Schluss, dass die Aufforderung, die Milch selbst zu holen, im Falle von Odiles Äußerung das Gesagte ist. Man könnte sich fragen, wo dann bei Baz die Unterscheidung zwischen Implikaturen und dem Gesagten überhaupt eine Rolle spielt. In diesem Kontext sollten wir uns allerdings vor Augen halten, dass Baz offenlässt, in welchem Umfang das, was er über den Begriff des Wissens sagt, auf andere Begriffe übertragbar ist. ${ }^{57}$ Bisweilen lässt Baz anklingen, dass der Einfluss, den der Witz einzelner Äußerungen von Sätzen, die das Wort »wissen « enthalten, einen größeren Einfluss auf deren Bedeutung hat, als es bei anderen Ausdrücken der Fall ist. Es ist anzunehmen, dass Baz beispielsweise bei Grices berühmtem Handschriften-Fall zustimmen würde, dass der Professor nicht sagt, sondern implikiert, dass sein Student kein guter Philosoph ist. ${ }^{58}$

Das Beispiel um Odile und Hugo ist aber nicht der einzige Kontext, in dem bei Baz eine radikale Position hinsichtlich der Flexibilität der Bedeutung von »wissen« anklingt. Eine weitere einschlägige Passage bezieht sich auf den

$5^{6}$ Baz 2012, S. 120. Angesichts dieses Zugeständnisses ist Baz' wiederholte Stellungnahme gegen die Unterscheidung zwischen Semantik und Pragmatik erläuterungsbedürftig. Vgl. etwa Baz 2009, S. 201, Baz 2012, S. 138.

57 Häufig verwendet er in seinen Behauptungen über das Funktionieren sprachlicher Ausdrücke die Qualifikation: »at least in the case of `know that « and its cognates«. Vgl. z. B. Baz 2012, S. 105, 118, 128, 200. In The Crisis of Method in Contemporary Analytic Philosophy führt Baz weitere Beispiele für sprachliche Ausdrücke an, die nicht ohne Weiteres »auf Fälle angewendet« werden können: »think«, »cause «, »free«, »mean«, »see«, »morally responsible«, »understand«, »intentional« und »pain« (Vgl. Baz 2017, S. 71f., 76, 78, 87, 129, 146).

$5^{8}$ Das Beispiel lautet wie folgt: »I am reporting on a pupil at Collections. All I say is >Jones has beautiful handwriting and his English is grammatical.< We might perhaps agree that there would here be a strong, even overwhelming, implication that Jones is no good at philosophy« (Grice 1961, S. 129). Vgl. dazu auch das Kapitel 4 des vorliegenden Buchs. 
oben bereits erwähnten Flughafen-Fall von Stewart Cohen. Zur Erinnerung: Die Protagonisten dieses Falls bekommen mit, wie der Passagier Smith gefragt wird, ob er weiß, ob ein bestimmter Flug einen Zwischenaufenthalt in Chicago hat. Smith schaut auf seinen Flugplan und bejaht die gestellte Frage: »Yes, I know - it does stop in Chicago.« In Baz' Diskussion dieses Beispiels lesen wir:

Passenger Smith's 'Yes, I know' in this story is more or less natural or anyway not clearly unnatural; but it is natural precisely because, or insofar as, it means something like 'Yes, I happen to have the information you're asking for', or 'Yes, my itinerary contains the information you need'. His 'Yes I know', uttered as he is looking at his itinerary, is most reasonably understood as a signal that he has found or identified the relevant information on his itinerary. ${ }^{59}$

Anscheinend dürfen wir Baz hier wörtlich nehmen: Er geht davon aus, Smiths Äußerung von »Yes, I know« könne bedeuten, dass er die erfragte Information auf dem Reiseplan gefunden habe. Auf die oben zitierte Passage folgen nämlich direkt die folgenden Sätze:

Only prior commitment to the assumption that 'know that' and its cognates have a 'semantic content' that is separable from the different types of work they ordinarily and normally do for us would lead one to insist that this is something that Smith is merely implicating with his 'I know' while strictly and literally saying something else. Give up the commitment, and talk of 'implicature' would seem forced and unmotivated in this case. ${ }^{60}$

Baz zufolge haben wir keinen Grund dafür, darauf zu bestehen, dass Smith nicht wirklich sagt, dass sein Reiseplan die benötigte Information enthalte. Eine solche Behauptung liegt, so Baz' Idee, nur für eine Person nahe, die irrtümlicherweise annimmt, man könne die Bedeutung des Ausdrucks »wissen« von der Arbeit trennen, die dieses Wort üblicherweise leistet. Diese Bemerkung ist prima facie ungereimt. Wenn sich die Bedeutung des Ausdrucks »wissen« aus der Arbeit ergibt, die dieses Wort üblicherweise leistet, dann gibt es doch eben eine Standardbedeutung von »wissen « und eine Standardbedeutung von »I know«, die nichts mit einem Fahrplan oder anderen Aspekten der spezifischen, oben beschriebenen Äußerungssituation zu tun haben!

Damit Baz' Bemerkung überhaupt Sinn ergibt, müssen wir seine Position zunächst einmal so verstehen, dass sie sich nicht auf die Bedeutung von »wissen « im Allgemeinen bezieht, sondern auf die Bedeutung einzelner, spezifischer Satz-Konstruktionen, die das Wort »wissen« enthalten, zum

59 Baz 2012, S. $172 f$.

6o Baz 2012, S. 173 . 
Beispiel eben auf den spezifischen Ausdruck»I know « oder »Yes, I know«. Die Standardbedeutung dieses Satzes hängt davon ab, wofür genau dieser Ausdruck üblicherweise verwendet wird, und muss deshalb unabhängig davon bestimmt werden, was beispielsweise die im Fall um Odile und Hugo verwendete Konstruktion »You know< + Interrogativsatz« bedeutet. Dann ist Baz' Idee offenbar, dass einer der üblichen Zwecke der Verwendung von »(Yes,) I know « darin besteht, zum Ausdruck zu bringen, dass mir eine erfragte Information in irgendeiner Weise vorliegt. Und wenn ich bei der Äußerung des Satzes meinen Fahrplan betrachte, dann ist auch klar, in welcher Weise mir die Information vorliegt, und ich bringe mit der Äußerung von »Yes, I know - it does stop in Chicago« zum Ausdruck: Meinem Fahrplan zufolge hat der Flug einen Zwischenaufenthalt in Chicago.

Analog dazu muss Baz' Idee im Fall um Odile und Hugo sein, dass es einer der üblichen Zwecke der Verwendung von »YYou know< + Interrogativsatz« ist, jemanden zu etwas aufzufordern. Unabhängig davon, wie verbreitet diese Nutzung der Konstruktion »YYou know< + Interrogativsatz« ist, scheint mir Baz' Auffassung gerade in Bezug auf dieses Beispiel verfehlt. Die Bedeutung, die der Satz »Du weißt, wo die Milch ist « einer traditionellen Analyse zufolge von der Art, wie sie Baz ablehnt, hat, geht offensichtlich in die Bedeutung von Odiles Äußerung ein: Odile führt gleichsam einen Grund dafür an, weshalb es nicht nötig ist, dass sie die Milch für Hugo holt: Er kann sie selbst holen, denn er weiß, wo sie ist. Dass Odiles Äußerung im Wortsinn keine Aufforderung ist, sieht man weiterhin daran, dass sie einen Wahrheitswert hat. Hugo reagiert auf Odiles Äußerung nicht mit einer Entgegnung, die in die Richtung geht von: »Woher sollte ich es wissen, ich bin schließlich das erste Mal bei dir zu Besuch!«, sondern ersinnt abwegige Alternativen zum für ihn anscheinend offensichtlichen Standort der Milch: »Perhaps the cat broke into the refrigerator, or there was just now a very stealthy milk thief, or it evaporated or suddenly congealed.« Angenommen, dass sich die Milch wie erwartet im Kühlschrank befindet, und vorausgesetzt, dass wir die im gegebenen Kontext üblichen Standards für Wissen ansetzen, sagt Odile also etwas Wahres: Hugo weiß tatsächlich, wo die Milch ist. Und würde Odile plötzlich einfallen, dass Hugo eigentlich noch nie bei ihr in der Wohnung war und der Kühlschrank nicht leicht zu finden ist, wäre es adäquat, wenn sie sagen würde: »Ach nein, du weißt es ja gar nicht!«. Dies entspräche nicht nur syntaktisch, sondern auch semantisch der Negation ihrer vorangehenden Äußerung. Mit seinen Vorstellungen von der Variabilität in der Bedeutung von Sätzen, die das Wort »wissen« enthalten, geht Baz mithin zu weit.

Wie ist es mit Ernsts Position zur Mehrdeutigkeit von » $S$ weiß, dass $p \ll$ ? Dass wir manchmal Fragen der Form »Weiß $S$, dass $p$ ? « stellen, ohne uns dafür zu 
interessieren, welche Anhaltspunkte $S$ für $p$ hat beziehungsweise ob die Überzeugung von $S$, dass $p$, gegebenenfalls gerechtfertigt ist, ist offensichtlich. Ich habe weiter oben bereits den Fall erwähnt, in dem sich jemand erkundigt, ob seine Bürokollegin weiß, dass eine Sitzung verschoben wurde. Ein anderer Fall sind Prüfungssituationen: Wenn ein Lehrer sich aus Gründen der Statistik bei einem anderen Lehrer erkundigt: »Wie viel Prozent der Schüler haben die Antwort auf Frage 4 gewusst?«, dann interessiert er sich nicht für den Unterschied zwischen Schülern, die sich ihrer Sache sicher waren, und solchen, die die richtige Antwort nur erraten haben. Die Interessen, aus denen eine Frage gestellt wird, haben aber nicht unbedingt einen Einfluss auf die Bedeutung der Frage. Alles bisher Gesagte wäre mit der Auffassung vereinbar, dass es korrekt wäre, die Frage nach der Bürokollegin mit »Nein « zu beantworten, wenn Letztere keine geeignete Rechtfertigung für ihre Überzeugung hat, dass die Sitzung verschoben wurde, und dass wir an der Zahl der richtigen Antworten auf Frage 4 nicht ablesen können, wie viele Schüler diese Antwort gewusst haben.

Dafür, dass manchmal für die korrekte Zuschreibung von Wissen in der Tat eine wahre Überzeugung hinreichend ist, argumentiert Ernst in »Two Varieties of Knowledge « anhand von zwei Gedankenexperimenten, beides Variationen des bekannten Scheunen-Beispiels von Alvin Goldman. Ernst gibt dieses Beispiel wie folgt wieder:

Ein Mann, nennen wir ihn »Barney«, fährt auf der Landstraße und schaut aus dem Fenster seines Autos. Er sieht eine Scheune und kommt zu der Überzeugung, daß er soeben an einer Scheune vorbeigefahren ist, und tatsächlich ist er auch an einer Scheune vorbeigefahren. Was Barney jedoch nicht weiß, ist, daß die Bewohner des Landstrichs (aus welchen Gründen auch immer) zahlreiche Scheunenattrappen (Potemkinsche Scheunen) an der Landstraße aufgestellt haben, die vom Auto aus nicht als Attrappen zu erkennen sind. Barney hatte aber Glück und hat die einzige echte Scheune im Umkreis gesehen. In diesem Fall hat Barney also gerechtfertigterweise eine wahre Überzeugung, und doch würden wir nicht sagen, er wisse, daß er an einer Scheune vorbeigefahren ist. ${ }^{61}$

Ernst zufolge wird durch diese Beschreibung des Falls nahegelegt, Barney als potentiellen Informanten zu beurteilen, deshalb sind wir dazu geneigt, ihm Wissen abzusprechen. Ernsts Variationen des Falls legen demgegenüber nahe, Barney als Akteur zu beurteilen:

61 Ernst 2002, S. 2of. Die ursprüngliche Beschreibung des Falls findet sich in Goldman 1976, S. $772 f$. 
Variation 1: Ich entdecke auf einem Feld eine Goldmine und beschließe, das entsprechende Stück Land zu kaufen, sobald ich genug Geld dafür habe. Wo die Goldmine sich befindet, kann ich mir gut merken, da an der betreffenden Stelle die einzige Scheune im Umkreis vieler Kilometer steht. Bevor ich das Geld für den Kauf des Landes ansparen kann, findet leider mein wohlhabender Erzfeind Barney heraus, dass ich eine Goldmine entdeckt habe, die sich unter der einzigen Scheune weit und breit befindet, und macht sich auf die Suche nach dieser Scheune. Um ihm das Finden der Goldmine zu erschweren, lasse ich an verschiedenen Stellen im weiteren Umkreis Scheunenattrappen aufstellen, was Barney nicht weiß. Bevor Barney irgendeine der Attrappen sieht, fährt er mit seinem Auto an der einzigen echten Scheune vorbei und bildet die wahre Überzeugung, dass an der Stelle, wo die Scheune steht, die Goldmine vergraben ist. Weiß Barney, wo die Goldmine vergraben ist?62

Variation 2: Barney ist ein Kind und spielt gerade draußen, als es heftig zu regnen beginnt. In dem ländlichen Gebiet, in dem sich Barney befindet, gibt es neben zahlreichen Scheunenattrappen eine einzige echte Scheune, an der Barney gerade zu dem Zeitpunkt vorbeikommt, an dem es zu regnen beginnt. Von den Scheunenattrappen weiß Barney nichts. Statt Unterschlupf in der Scheune zu suchen, spielt Barney im Regen weiter und kommt später völlig durchnässt und mit einer Erkältung nach Hause. Seine Mutter ärgert sich darüber, dass Barney sich nicht in der Scheune vor dem Regen geschützt hat. Barney hat mittlerweile von den Scheunenattrappen erfahren und versucht, sich zu verteidigen: »Aber ich wusste doch gar nicht, dass ich an einer Scheune vorbeikam!«. Wusste Barney wirklich nicht, dass er an einer Scheune vorbeikam?63

Ernst schreibt in Bezug auf beide Variationen: »[F]or all intents and purposes Barney knows [or: knew] that there is [or: that there was] a barn. «64 Man könnte stattdessen aber auch sagen: Ob Barney weiß oder nicht weiß (bzw. wusste oder nicht wusste), dass an der betreffenden Stelle eine Scheune steht (bzw. stand), ist für die jeweiligen Zwecke einfach irrelevant. In Variation 1 ist relevant, dass Barney die Goldmine finden und damit die Absichten ihrer ursprünglichen Entdeckerin vereiteln wird. In Variation 2 ist relevant, dass wir

\footnotetext{
62 Vgl. Ernst 2012, S. $320 f$.

63 Vgl. Ernst 2012, S. 321. Vgl. für einen ähnlichen Fall auch Hanfling 2003.

64 Ernst 2012, S. 321. Man beachte allerdings, dass Ernst nicht der Meinung ist, dass es in den betreffenden Szenarien falsch wäre, Barney Wissen abzusprechen: Die Beschreibungen legen zwar nahe, Barney als Akteur zu beurteilen und somit zu entscheiden, ob die objektive Variante von Wissen vorliegt, aber natürlich kann man Barney auch in den Variationen 1 und 2 stattdessen als potentiellen Informanten beurteilen und entscheiden, ob die perspektivische Variante von Wissen vorliegt oder nicht (vgl. Ernst 2012, S. 321f.).
} 
Barney dafür kritisieren können, dass er nicht in der Scheune Unterschlupf gesucht hat, die er gesehen hat (ohne im Geringsten daran zu zweifeln, dass es eine Scheune war). Für beide Fälle ist es unerheblich, ob Barney in Bezug auf den Standort der (bzw. einer) Scheune über Wissen verfügt(e).

Tatsächlich widerspricht es angesichts der Existenz von Scheunenattrappen zumindest meinem Sprachgefühl, über den Regen-Barney zu behaupten, dass er wusste, dass er eine Scheune vor sich hatte. Er hatte eine wahre Überzeugung, und das ist ausreichend dafür, dass man ihn dafür kritisieren kann, sich nicht in der Scheune vor dem Regen geschützt zu haben.

Etwas anders sieht es in der Variation 1 aus. Dort würde der ursprüngliche Entdecker der Scheune in der Tat nichts Unwahres sagen, sollte er ausrufen: »Na toll - jetzt weiß Barney, wo die Goldmine ist!«. Mit einem Beleg dafür, dass für Wissen in bestimmten Fällen eine wahre Überzeugung hinreichend ist, haben wir es hier jedoch meines Erachtens trotzdem nicht zu tun. Wenn ich in einem Strategie-Spiel einen unüberlegten Zug mache und dann enttäuscht bemerke: »Na toll - jetzt hast du gewonnen«, dann sage ich damit auch nichts Falsches, aber gemeint ist damit: Jetzt steht fest, dass du gewinnen wirst. Und ebenso könnte im Goldminen-Fall der oben formulierte Ausruf paraphrasiert werden mit: »Na toll - jetzt steht fest, dass Barney wissen wird, wo die Goldmine ist!«. Und zwar steht es deswegen fest, weil Barney an der richtigen Stelle graben und die Goldmine finden wird.

Ernst würde vermutlich entgegnen, dass mein Urteil, dass der RegenBarney und der Goldminen-Barney nicht über Wissen verfügen, darauf beruht, dass ich zu den beiden Fällen eine andere Perspektive als die von ihm vorgeschlagene eingenommen und die Barneys als mögliche Informanten statt als Akteure beurteilt habe. Aber mir ergibt sich aus keiner Perspektive, die es mir einzunehmen gelingt, der Eindruck, dass es für die Wahrheit der Behauptung, Barney wisse, dass er eine (echte) Scheune sehe, hinreichend ist, dass er eine wahre Überzeugung hat. Das muss natürlich nicht heißen, dass Ernst mit seinen Ausführungen zu den Bedingungen, unter denen Akteure über Wissen verfügen, falsch liegt. Es kann stattdessen auch so sein, dass Ernst das Wort »wissen « anders verwendet als ich und demzufolge einen geringfügig anderen Wissensbegriff besitzt als ich.

Ich habe keine grundsätzlichen Vorbehalte dagegen, einzuräumen, dass »wissen« je nach Kontext Unterschiedliches bedeutet. Hanflings Unterscheidung von Situationstypen, in denen wir das Wort »wissen « verwenden, legt bereits dergleichen nahe. ${ }^{65}$ Ich schließe also nicht prinzipiell aus, dass

65 Diese Unterscheidung wurde zu Beginn des Kapitels 2 des vorliegenden Buchs rekonstruiert. 
ich es als adäquat empfinden würde, über Akteure in bestimmten Situationen auch dann zu sagen, sie wüssten, dass soundso, wenn keine geeignete Rechtfertigung für die betreffenden wahren Überzeugungen vorliegt. Sollte es Fälle geben, die sich vor dem Hintergrund meines Sprachgebrauchs als Belege für eine solche zweite Variante von Wissen nicht so leicht weg-erklären lassen wie Ernsts Variationen des Barney-Beispiels, dann gebe ich gern zu, dass Ernst Recht hat. Die Fälle, die er in »Two Varieties of Knowledge« bespricht, überzeugen mich jedoch nicht.

\section{7} Schluss

Ich habe im vorliegenden Kapitel dafür argumentiert, dass die Frage des Epistemologen contra Baz eine Bedeutung hat. Die Bedeutung, die eine Frage der Form »Weiß $S$, dass $p$ ? « im Kontext eines epistemologischen Gedankenexperiments hat, lässt sich wie folgt ausbuchstabieren: »Wäre es angesichts der im Gedankenexperiment beschriebenen Umstände korrekt, über $S$ zu sagen, dass er oder sie weiß, dass $p ?$ «. $^{66}$ Diese Frage könnte sich für uns contra Baz auch in einem alltäglichen Gettier-Fall stellen. Zum Beispiel wenn wir als informierte Betrachterinnen oder Betrachter von einer weniger gut informierten Person gefragt werden, ob $S$ wisse, ob $p$, oder wenn wir uns als Biografin oder Biograf von $S$ für eine bestimmte Formulierung entscheiden müssen. Da Baz’ Argument dafür, dass unseren Reaktionen auf die Frage des

66 Gegen die Auffassung, dass die Frage »Weiß $S$, dass $p$ ?«, bezogen auf epistemologische Gedankenexperimente, anders als etwas in einem Fall, in welchem ich mich danach erkundige, ob meine Bürokollegin weiß, dass die Sitzung verschoben wurde, eine $B e-$ schreibungsfrage ausdrückt, hat Hans-Johann Glock in einer Diskussion Folgendes eingewendet: Dass die Frage des Epistemologen anders als die Frage betreffend die Terminverschiebung ausschließlich auf der Grundlage von Sprachkompetenz, und nicht unter Einbezug von empirischen Informationen zu beantworten ist, beeinflusst nicht die Bedeutung der Frage. Dies lässt sich, so Glock, anhand der folgenden Analogie deutlich machen: In einem Revisionsverfahren im Gegensatz zu einem Berufungsverfahren werden keine neuen Beweise gesammelt oder berücksichtigt, sondern es wird allein auf der Grundlage der bereits in der früheren Verhandlung zugelassenen Beweise ein frisches Urteil gefällt. Trotzdem bedeutet beispielsweise die Frage: »Hat sich N. N. des Diebstahls schuldig gemacht? « dasselbe, egal, ob sie in einem Revisionsverfahren oder in einem Berufungsverfahren beantwortet wird. Ich bin allerdings nicht überzeugt, dass der Fragesatz »Hat sich N. N. des Diebstahls schuldig gemacht?« im Kontext eines Revisionsverfahrens seine übliche Bedeutung hat. Denn wenn zum Beispiel eine Videoaufnahme, die bisher noch nicht verfügbar war und die N. N. zum angeblichen Tatzeitpunkt zeigt, für den für das Revisionsverfahren verantwortlichen Richter nicht relevant ist, ist die Frage, die er beantworten will, offenbar nicht wirklich, ob sich N. N. des Diebstahls schuldig gemacht hat. 
Epistemologen kein Gewicht für die Analyse unseres Wissensbegriffs zukommt, auf der Prämisse beruht, dass sich die Frage des Epistemologen im Alltag nicht stellt, ist sein Argument nicht schlüssig.

Wie verhält es sich aber tatsächlich mit unserer Kompetenz in der Beantwortung der Frage des Epistemologen? Gerade in Bezug auf epistemologische Gedankenexperimente, die besonders exotisch und komplex sind, kommt es doch tatsächlich vor, dass wir mit der Entscheidung, ob in den betreffenden Fällen Wissen vorliegt oder nicht, überfordert sind. In diesem Kontext sind meines Erachtens zwei Punkte besonders relevant. Erstens: Es gibt keine Fakten über die richtige Beantwortung der Frage des Epistemologen, die uns als kompetente Sprecherinnen und Sprecher nicht zugänglich sind. Zweitens: Bezogen auf bestimmte philosophische Gedankenexperimente kann die Frage »Weiß $S$, dass $p$ ? « vielleicht tatsächlich nicht korrekt mit Ja oder Nein beantwortet werden. Auch die Erkenntnis, dass die Regeln zum Gebrauch des Ausdrucks »wissen « in einem bestimmten Fall nicht festlegen, ob Wissen vorliegt oder nicht, ist aber für die Analyse unseres Wissensbegriffs hilfreich. ${ }^{67}$ Auf diese beiden Punkte werde an anderen Stellen des vorliegenden Buchs zurückkommen - unter anderem im Zusammenhang mit der Experimentellen Philosophie.

67 Vgl. Ernst 2015, S. 182. 